\title{
Como Avaliar o Risco e Prescrever Exercício no Cardiopata
}

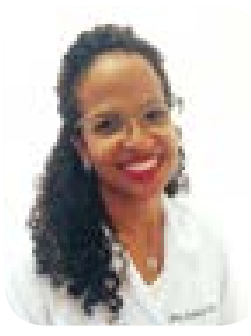

Atividade física no paciente com doença cardiovascular (DCV) tem sido demonstrada a promover: aumento da capacidade funcional, redução ou eliminação de angina, aumento de distância percorrida em pacientes com claudicação, redução de morte por todas as causas e por causa cardiovascular e redução de reinternações ${ }^{1,2}$.

Mas, apesar de todos esses benefícios, a atividade física não é prescrita de forma consistente para os cardiopatas, o que é evidenciado pelo fato de que apenas 14 a $35 \%$ dos pacientes são referenciados para programas de reabilitação após um infarto e após uma cirurgia de revascularização miocárdica ${ }^{3}$. Um dos motivos é que alguns clínicos subestimam ou desconhecem os benefícios da reabilitação e outros superestimam as taxas de complicações.

O risco de complicações cardíacas é muito baixo em programas de reabilitação: estima-se que o risco de ocorrer uma parada cardíaca, morte ou infarto seja de 1 evento por cada 60.000 - 80.000 horas de exercício supervisionado 4 . E essa baixa incidência está relacionada a uma adequada estratificação de risco cardiovascular pré-participação.

\section{AVALIAÇÃO DE RISCO}

Os indivíduos podem ser classificados por risco com base na sua idade e características dos fatores de risco cardíaco. A American Heart Association sugere esta classificação apresentada a seguir, que é utilizada para determinar a necessidade de uma supervisão médica durante o exercício e monitoramento necessário ${ }^{1}$.

Os indivíduos da Classe A são os aparentemente saudáveis. A maioria não precisa de avaliação médica para participar de atividade física de leve intensidade. Entre os homens $<45$ anos e as mulheres $<55$ anos de idade que são assintomáticos, sem doença cardiovascular conhecida ou suspeita, a avaliação médica pré-participação não é geralmente necessária, a menos que existam circunstâncias agravantes, como uma história familiar de morte súbita em idade jovem ou fatores de risco cardíaco mal controlados. Entre os homens> 45 anos e as mulheres> 55 anos de idade que pretendem realizar exercício vigoroso, que sejam portadores de diabetes mellitus ou outros 2 fatores de risco para DCV, é recomendada uma avaliação médica. Esta deve incluir anamnese, exame físico e teste ergométrico (TE) ou ergoespirometria ${ }^{1}$.

Os indivíduos da classe B são os portadores de doença cardiovascular estável, com baixo risco de complicações no exercício. Esta classificação inclui indivíduos com qualquer dos seguintes diagnósticos: doença arterial coronariana (DAC) [infarto agudo do miocárdio (IAM), cirurgia de revascularização do miocárdio, angioplastia, angina pectoris]; doença cardíaca valvular, excluindo estenose valvar ou regurgitação grave; doença cardíaca congênita (a estratificação de risco para pacientes com cardiopatia congênita deve ser guiada pela $27^{a}$ Conferência de Bethesda 5); cardiomiopatia [inclui pacientes estáveis com insuficiência cardíaca (IC) com características clínicas, conforme descrito abaixo, mas não cardiomiopatia hipertrófica ou miocardite recente] $]^{1}$.

- Características clínicas (devem incluir todas as seguintes):

- Classe Funcional I ou II (NYHA - New York Heart Association);

- Capacidade funcional > 6 METs (observada em teste ergométrico);

- Sem evidência de IC descompensada;

- Sem angina ou isquemia no teste ergométrico $\leq$ 6 METs (baixa carga de esforço);

- Aumento adequado da pressão arterial sistólica durante TE;

- Ausência de taquicardia ventricular (TV) em repouso ou exercício.

Para esse perfil de paciente, indica-se exercício sob supervisão médica (em um Centro de Reabilitação Cardíaca) ou exercício não supervisionado, em uma academia. A reabilitação cardíaca em uma unidade especializada não é obrigatória para indivíduos da classe 
$B$, ela é sugerida como benéfica, durante as sessões iniciais, porque exercício vigoroso pode ser conduzido de forma mais segura ${ }^{1}$.

Os indivíduos que fazem parte da classe $\mathrm{C}$ são aqueles que apresentam moderado a alto risco de complicações durante exercício, com qualquer um dos seguintes:

- Classe Funcional III ou IV (NYHA);

- Capacidade funcional < 6 METs (TE);

- Angina em repouso;

- Angina ou infradesnivelamento de segmento ST isquêmico no TE < 6METs;

- Queda da pressão arterial sistólica durante exercício para valores menores que os de repouso;

- TV não sustentada no exercício;

- Parada cardiorrespiratória prévia (que não tenha ocorrido durante IAM ou durante procedimento cardíaco).

Para os pacientes da classe $\mathrm{C}$, deve haver supervisão médica durante todas as sessões de exercício até que a segurança seja estabelecida ${ }^{1}$.

Pacientes estáveis podem ser encaminhados à reabilitação 1 ou 2 semanas após alta hospitalar, após evento cardíaco, como IAM ou angioplastia'. O exercício não supervisionado também pode ser usado para o condicionamento após o indivíduo se recuperar do IAM ( $\geq 2$ semanas após a alta hospitalar) ou em outros casos de DAC estável ${ }^{6}$, embora o exercício sob supervisão médica seja preferível. Pacientes com DAC que tenham sido submetidos à cirurgia cardíaca recente devem ser examinados, observando-se infecções de feridas e instabilidade esternal para garantir que eles não se envolvam em atividades físicas que iriam prejudicar a cicatrização. Para esses pacientes, é amplamente aceito que deve haver um atraso no início de um programa de treinamento de resistência de extremidade superior após a cirurgia (isto é, 4-6 semanas) ${ }^{1}$.

Evidências recentes indicam que o início de uma reabilitação cardíaca estruturada logo após a alta hospitalar para um IAM recente melhora significativamente a adesão no programa ${ }^{7}$. Os clínicos devem, portanto, considerar fortemente o encaminhamento para reabilitação cardíaca logo que possível, após a alta hospitalar. Se não houver instalações de reabilitação cardíaca disponíveis, a prescrição de atividade ainda pode ser fornecida a pacientes cardiopatas e eles devem ser encorajados a exercitar-se.

A maioria dos indivíduos, com a orientação de seu médico, pode iniciar um programa de caminhada em casa em um ritmo lento e regular, com duração crescente, começando com um a dois períodos de 5 a 10 minutos por dia e aumentando gradualmente até 30 a 60 minutos por dia. Se os indivíduos observam atentamente sinais de intolerância, tais como dor no peito ou falta de ar e estão atentos à frequência cardíaca e à percepção subjetiva de esforço, este nível de atividade é considerado seguro. Caminhada é um exercício seguro, de baixo impacto, controlável, que, na maioria dos casos, gera uma intensidade que é de $40 \%$ a $70 \%$ do $\mathrm{VO}_{2}$ máximo'.

Por fim, os indivíduos da classe $D$ são aqueles com doença cardíaca instável, com restrição à prática de exercício. Esta classificação inclui indivíduos com qualquer um dos seguintes: angina instável, estenose ou insuficiência valvar grave e sintomática, IC descompensada, arritmias não controladas. Nenhuma atividade é recomendada para fins de condicionamento. A atenção deve ser direcionada ao tratamento e à restauração do paciente para a classe $C$ ou $B$. As atividades diárias devem ser prescritas com base na avaliação individual do médico assistente ${ }^{1}$.

\section{COMO PRESCREVER EXERCÍCIO}

A reabilitação deve ser composta por exercícios aeróbicos, de resistência e de flexibilidade ${ }^{1,8}$. Neste artigo será abordada apenas a prescrição aeróbica.

Os objetivos do treinamento ideal são exercitar-se $\geq$ 5 dias por semana, durante 30 a 60 minutos, sendo caminhada, bicicleta ou esteira ergométrica ${ }^{1,9}$. No entanto, essa frequência de atividade pode ser demasiada para alguns, particularmente aqueles que estão frágeis ou descondicionados no início. Portanto, é importante começar com metas modestas, que sejam realistas e alcançáveis, e avançar lentamente para a intensidade, duração e frequência desejáveis.

Cada sessão é composta por 3 componentes: aquecimento, treinamento propriamente dito e desaquecimento, nesta ordem (figura 1). Ambos os períodos de aquecimento e desaquecimento envolvem exercícios aeróbicos de baixa intensidade normalmente por 5 a 10 minutos. As manobras de aquecimento estimulam a vasodilatação e aumentam a perfusão muscular local antes de um estímulo mais intenso. Essa atividade também aumenta a amplitude de movimento articular e a flexibilidade ${ }^{1,9}$.

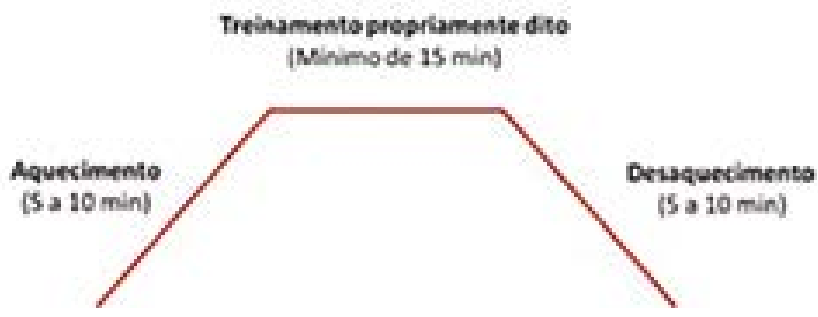

Figura 1 - Componentes de cada sessão aeróbica. 
Após o treino propriamente dito, o desaquecimento facilita a transição gradual para o término do exercício, modulando os efeitos da vasodilatação e da elevação de catecolaminas. Essa volta à calma conduz à transição gradual da frequência cardíaca e da pressão arterial para os níveis de repouso, reduzindo a probabilidade de hipotensão e ectopia ventricular ${ }^{1}$.

Habitualmente se começa com um treinamento de curta duração (15 minutos) durante a primeira sessão e com uma progressão semanal ou por sessão, de acordo com o caso ${ }^{8}$. A intensidade do treinamento deve ser controlada pela frequência cardíaca de treinamento (FCT). Nesta estratégia, busca-se realizar os exercícios prescritos entre $40 \%$ e $80 \%$ da frequência cardíaca de reserva (FC máxima - $F C$ repouso), utilizando a FC máxima alcançada no teste de exercício, através da fórmula de Karvonen ${ }^{1,8}$.

Fórmula de Karvonen: $\mathrm{FCT}=\mathrm{FC}$ repouso $+(0,4 \mathrm{a}$ $0,8) \times(F C$ máxima $-F C$ repouso).

Por exemplo, se a FC máxima for 180 e a FC em repouso for de 80 , a $\mathrm{FC}$ de reserva é 100 . Treinando a $50 \%$ da FC de reserva, a FCT inicial será130 bpm (80 $+0,5 \times 100$ ).

O teste cardiopulmonar de exercício ou ergoespirometria fornece a medida mais precisa do desempenho aeróbio máximo durante um teste de exercício ( $\mathrm{VO}_{2}$ max), de modo que a intensidade de treinamento possa ser estabelecida como uma porcentagem do $\mathrm{VO}_{2} \max ^{1}$. Por meio da análise conjunta dos gases expirados, do trabalho e/ou esforço realizado e do comportamento de variáveis hemodinâmicas, pode-se obter uma avaliação funcional mais completa. Dessa forma, é possível se delinear um programa de exercícios aeróbicos mais preciso e individualizado ${ }^{10}$. A intensidade do treinamento, utilizando-se a ergoespirometria, define a FCT entre 40 e $80 \%$ do $\mathrm{VO}_{2} \max$ (utilizando-se a FC atingida no $\left.\mathrm{VO}_{2} \max \right)^{1}$.

Para os indivíduos que cursam com dor precordial ou infradesnivelamento do segmento ST durante o teste ergométrico, os exercícios devem ser realizados abaixo do limiar isquêmico, ou seja, abaixo da FC e carga que levem à indução de sinais clínicos e/ou eletrocardiográficos de isquemia miocárdica no esforço ${ }^{8}$.

Como o nível de aptidão de um indivíduo avança, a intensidade do treinamento precisa avançar, portanto, é importante periodicamente reavaliar a prescrição do exercício com base no estado de aptidão e condicionamento atual do paciente ${ }^{1}$.

Em conclusão, a prescrição de exercício para o cardiopata não é tarefa exclusiva do cardiologista especialista em reabilitação cardíaca. O treinamento físico faz parte do arsenal do tratamento clínico otimizado, devendo fazer parte da prescrição rotineira do cardiologista clínico, além do tratamento farmacológico habitual.

\section{REFERÊNCIAS}

1. Fletcher GF et al. Exercise Standards for Testingand Training. A ScientificStatementFromthe American Heart Association. Circulation. 2013;128:873-934.

2. Heran BS, Chen JM, Ebrahim S, Moxham T, OIdridge N, ReesK, Thompson DR, Taylor RS. Exercise-basedcardiacrehabilitation for coronaryheartdisease. Cochrane DatabaseSyst Rev. 2016,Issue1.Art. No.:CD001800

3. Suaya JA, Shepard DS, Normand SL, Ades PA, Prottas J, Stason WB. Use ofcardiacrehabilitationbyMedicarebeneficiariesaftermyocardialinfarctionorcoronarybypasssurgery. Circulation. 2007;116:1653-1662.

4. Thompson PD, Franklin BA, Balady GJ, Blair SN, Corrado D, Estes NA et al. Circulation. 2007;115(17):2358-68.

5. Fuster V, Gotto AM, Libby P, Loscalzo J, McGill HC. 27thBethesdaConference: matchingtheintensityofriskfactor management withthehazard for coronarydiseaseevents. Task Force 1. Pathogenesisofcoronarydisease: thebiologic role ofriskfactors. J Am Coll Cardiol.1996;27:964-976.

6. Fletcher BJ, Lloyd A, Fletcher GF. Outpatientrehabilitative training in patientswith cardiovascular disease: emphasison training method. Heart Lung. 1988;17:199-205.

7. Parker K, Stone JA, Arena R, Lundberg D, Aggarwal S, GoodhartD, Traboulsi M. Anearlycardiacaccessclinicsignificantly improves cardiacrehabilitationparticipationandcompletion rates in low-risk ST-elevationmyocardialinfarctionpatients. Can J Cardiol. 2011;27:619-627.

8. Herdy AH, López-Jimenez F, Terzic CP, Milani $M$, Stein R, Carvalho T; Sociedade Brasileira de Cardiologia. Diretriz Sul-Americana de Prevenção e Reabilitação Cardiovascular. ArqBrasCardiol 2014; 103(2Supl.1): 1-31.

9. Shephard RJ, Balady GJ. Exercise as cardiovascular therapy.Circulation.1999;99:963-972.

10. Herdy AH et al. Teste Cardiopulmonar de Exercício: Fundamentos, Aplicabilidade e Interpretação. ArqBrasCardiol. 2016; 107(5):467-481

1- Serviço de Cardiologia Clínica do HSI

Endereço para correspondência:

isabelapilar@hotmail.com 